\title{
The Polyhedral Approach to the Maximum Planar Subgraph Problem: New Chances for Related Problems
}

\author{
Michael Jünger and Petra Mutzel \\ Universität zu Köln, Pohligstraße 1, 50969 Köln, Germany
}

\begin{abstract}
In [JM94] we used a branch and cut algorithm in order to determine a maximum weight planar subgraph of a given graph. One of the motivations was to produce a nice drawing of a given graph by drawing the found maximum planar subgraph, and then augmenting this drawing by the removed edges. Our experiments indicate that drawing algorithms for planar graphs which require 2- or 3-connectivity, resp. degreeconstraints, in addition to planarity often give "nicer" results. Thus we are led to the following problems:
\end{abstract}

(1) Find a maximum planar subgraph with maximum degree $d \in \mathbb{N}$.

(2) Augment a planar graph to a $k$-connected planar graph.

(3) Find a maximum planar $k$-connected subgraph of a given $k$ connected graph.

(4) Given a graph $G$, which is not necessarily planar and not necessarily $k$-connected, determine a new graph $H$ by removing $r$ edges and adding $a$ edges such that the new graph $H$ is planar, spanning, $k$-connected, each node $v$ has degree at most $D(v)$ and $r+a$ is minimum.

Problems (1), (2) and (3) have been discussed in the literature, we argue that a solution to the newly defined problem (4) is most useful for our goal. For all four problems we give a polyhedral formulation by defining different linear objective functions over the same polytope which is the intersection of the planar subgraph polytope [JM93], the $k$-connected subgraph polytope [S92] and the degree-constrained subgraph polytope. We point out why we are confident that a branch and cut algorithm for the new problem will be an implementable and useful tool in automatic graph drawing. 


\section{Introduction}

In [JM93] we defined the polytope $\mathcal{P} \mathcal{L S}(G)$ associated with all planar subgraphs of a given graph $G$. Here we define the polytope $k-\mathcal{N C S}(G)$ associated with all $k$-connected subgraphs of the graph $G$. Furthermore, we give a description of the integer points by a system of linear inequalities for both polytopes $\mathcal{P} \mathcal{L S}(G)$ and $k-\mathcal{N C S}(G)$. By intersecting the polytopes $\mathcal{P} \mathcal{L S}(G)$ and $k-\mathcal{N} \mathcal{C S}(G)$, we get a new polytope $k-\mathcal{N C P} \mathcal{L S}(G)$. We will see that by optimizing linear objective functions over the polytope $k-\mathcal{N C P} \mathcal{L S}(G)$, we will be able to solve the problem of finding a maximum planar $k$-connected subgraph $(k \geq 2)$ and the planar $(k$-)augmentation problem.

Both problems have applications in automatic graph drawing [TBB88]. In automatic graph drawing a given graph has to be layed-out in the plane in a nice and pleasant way. Nice drawings for sparse nonplanar graphs can be achieved by determining a maximum planar subgraph and augmenting an embedding of this graph. There are many layout algorithms which only work for planar graphs and/or $k$-connected graphs, where $k=2$ or $k=3$. Furthermore, there are drawing algorithms such as the minimizing bends algorithm of Tamassia [T87], that restrict the degrees of the nodes to a fixed number $d \in \mathbb{N}$. The degreeconstrained maximum planar subgraph problem is the problem of determining a maximum planar subgraph in which all nodes have degrees at most $d \in \mathbb{N}$.

The maximum planar $k$-connected subgraph problem is the task of finding a maximum planar subgraph of a given graph which is at least $k$-connected. Of course, this problem is only meaningful, if the given graph is at least $k$-connected. In this case, it is common to first determine a maximum planar subgraph and then solve the planar $k$-augmentation problem, which is the task of adding the minimum number of edges to a given planar graph in order to achieve a planar $k$-connected graph.

The natural problem arising in this context would be the following. Given a graph $G$, which is not necessarily planar and not necessarily $k$-connected, determine a new graph $H$ by removing $r$ edges and adding $a$ edges such that the new graph $H$ is planar, spanning, $k$-connected and $r+a$ is minimum. This problem never occurs in the literature before, we will call it the minimum planarizing $k$-augmentation problem. Adding the degree-constraints, saying that each node $v$ has degree at most $D(v)$, leads to the minimum planarizing degree-constrained $k$-augmentation problem. With the methods of polyhedral combinatorics, we can easily formulate all problems and it turns out that the new problem has the most natural formulation.

Indeed, it is advantageous to solve the minimum planarizing $k$-augmentation problem in comparison to the following approaches. (Note, that $k \in\{2,3\}$, whenever we talk about $k$-connectivity in context with planarity.)

(1) First solve the maximum planar subgraph problem and then solve the planar $k$-augmentation.problem on the detected planar subgraph. 


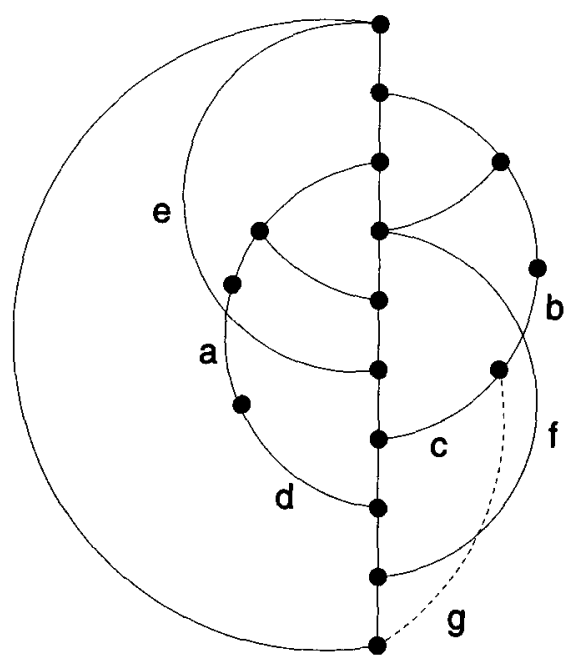

Fig. 1 .

(2) First augment the given graph to $k$-connectivity and then solve the $k$ connected planar subgraph problem.

For example, consider the graph $G$ given by the solid lines in Fig. 1. Approach (1) may lead to first removing the edges $a$ and $b$. In order to augment the graph to biconnectivity, we have to add at least three edges, whereas the optimum solution of the minimum planarizing 2-augmentation problem would be removing the edges $e$ and $f$. For the graph $G-\{c\}$, the second approach may lead to adding edge $c$, and then removing the edges $e$ and $f$, whereas the optimum solution of the minimum planarizing 2 -augmentation problem would be only to remove the edge $e$ and add the edge $g$.

The maximum planar subgraph problem is the following: Given a nonplanar weighted graph with edge weights $w_{e} \in \mathbb{R}$ for $e \in E$ we want delete a set of edges $F \subseteq E$ to obtain a planar subgraph $G^{\prime}=(V, E \backslash F)$ such that the sum of all edge weights $\sum_{e \in E \backslash F} w_{e}$ of $G^{\prime}$ is maximum. In the unweighted case, where $w_{e}=1$ for all edges $e \in E$, the problem consists of finding the minimum number of edges whose deletion from a nonplanar graph gives a planar subgraph. In either case the problem is NP-hard.

The (node-)connectivity $\kappa(G)$ of a graph $G$ is the minimum number of nodes whose removal together with its incident edges results in a disconnected or trivial graph. A graph is said to be $k$-node-connected, or $k$-connected, if $\kappa(G) \geq k$. The problem of augmenting a given graph by the minimum number of edges in order to obtain a $k$-connected graph seems still to be open, whereas there are polynomial time algorithms for $k=2$ and $k=3$ [RG77,HR91]. Frank gives a polynomial time algorithm for the $k$-edge-connected augmentation problem for arbitrary $k$ provided that the edge-costs are one (unweighted problem) or arise from node-costs, while the problem for arbitrary edge-costs is known to be NP-hard even for $k=2$ [F92]. The planar ( $k$-)augmentation problem has 
been brought up by Kant, and consists of adding a minimum number of edges to a planar graph in order to obtain a $k$-connected graph, which is still planar. Kant showed that this problem is NP-hard for $k=2$, and gives a linear time approximation algorithm which adds at most 2 times the minimum required number of edges [K93].

In [JM94], we attack the maximum planar subgraph problem with the techniques of polyhedral combinatorics. This approach gives us quite good and in many cases provably optimum solutions for sparse graphs and very dense graphs. Here we will see that it appears to be promising to use this approach also for the related problems discussed above.

In Sect. 2 of this paper we summarize results on the facial structure of the planar subgraph polytope given in [JM94] and [M94b]. In Sect. 3 we define the polytope associated to all $k$-connected spanning subgraphs of the graph $G$. Furthermore we give a system of linear inequalities characterizing the integer points in this polytope. In Sect. 4 we intersect both polytopes $\mathcal{P} \mathcal{L S}(G)$ and $k-\mathcal{N C S}(G)$. Using the new polytope, we show how to formulate the maximum planar $k$ connected subgraph problem and the planar $k$-augmentation problem. Moreover, we achieve a natural description of the newly defined minimum planarizing $k$ augmentation problem. Furthermore, we consider the degree-constraint versions of the problems. In Sect. 5 we suggest a "branch and cut" algorithm in order to solve the minimum planarizing 2-augmention problem. In Sect. 6 we give some computational results for the maximum planar subgraph problem and the degree-constrained maximum planar subgraph problem.

\section{The Planar Subgraph Polytope $\mathcal{P} \mathcal{L} \mathcal{S}(G)$}

Given a graph $G=(V, E)$ with edge weights $w_{e} \in \mathbb{R}$ for all $e \in E$, let $\mathcal{P}_{\mathcal{G}}$ be the set of all planar subgraphs of $G$. For each planar subgraph $P=\left(V^{\prime}, F\right) \in$ $\mathcal{P}_{\mathcal{G}}$, we define its incidence vector $\chi^{P} \in \mathbb{R}^{E}$ by setting $\chi_{e}^{P}=1$ if $e \in F$ and $\chi_{e}^{P}=0$ if $e \notin F$. The planar subgraph polytope $\mathcal{P} \mathcal{L}(G)$ of $G$ is defined as the convex hull over all incidence vectors of planar subgraphs of $G$. The problem of finding a planar subgraph $P$ of $G$ with weight $w(P)$ as large as possible can be written as the linear program $\max \left\{w^{T} x \mid x \in \mathcal{P C S}(G)\right\}$, since the vertices of the polytope $\mathcal{P} \mathcal{L S}(G)$ are exactly the incidence vectors of the planar subgraphs of $G$. Kuratowski characterized the minimal nonplanar graphs to be exactly the subdivisions of $K_{5}$ and $K_{3,3}$. Hence we get the following integer programming formulation for the maximum planar subgraph problem:

$$
\begin{aligned}
\text { maximize } & c^{T} x \\
\text { subject to } & 0 \leq x_{e} \leq 1, \quad \text { for all } e \in E, \\
& x(K) \leq|K|-1, \text { for all induced Kuratoski subgraphs } K \subseteq E \\
& x_{e} \text { integral, } \quad \text { for all } e \in E
\end{aligned}
$$

Since integer programming is NP-hard, we drop the integer constraints. In order to apply linear programming techniques to solve this linear program one 
has to represent $\mathcal{P} \mathcal{L S}(G)$ as the solution of an inequality system. Due to the NP-hardness of our problem, we cannot expect to be able to find a complete description of $\mathcal{P} \mathcal{L S}(G)$ by linear inequalities. But even a partial description of the facial structure of $\mathcal{P} \mathcal{L S}(G)$ by linear inequalities is useful for the design of a "branch and cut"-algorithm, because such a description defines a relaxation of the original problem. Such relaxations can be solved within a branch and bound framework via cutting plane techniques and linear programming in order to produce tight bounds. An irredundant description of $\mathcal{P} \mathcal{L S}(G)$ by linear inequalities contains only inequalities which describe proper faces of maximal dimension of $\mathcal{P} \mathcal{L}(G)$, so-called facet-defining inequalities. For efficiency, also in a partial description by inequalities, we concentrate on those valid inequalities for $\mathcal{P} \mathcal{L S}(G)$ which are facet-defining. For ease of notation, we define $x(F)=\sum_{e \in F} x_{e}$ for $F \subseteq E$. In [JM94] we state the following

Theorem 1 [JM94]. The dimension of the planar subgraph polytope $\mathcal{P} \mathcal{L S}(G)$ of $G=(V, E)$ is $|E|$, so it is full dimensional. For all edges $e \in E$ the inequalities $x_{e} \geq 0$ and $x_{e} \leq 1$ define facets of $\mathcal{P} \mathcal{L S}(G)$. For all subdivisions $G^{\prime}=\left(V^{\prime}, F\right)$ of $K_{5}$ or $K_{3,3}$ contained in $G$ the inequality $x(F) \leq|F|-1$ defines a facet of $\mathcal{P L S}(G)$. For all cliques $\left(V^{\prime}, F\right)$ (or complete bipartite subgraphs) contained in $G$, the Euler inequalities $x(F) \leq 3\left|V^{\prime}\right|-6$ (or $x(F) \leq 2\left|V^{\prime}\right|-4$, respectively) are facet-defining for $\mathcal{P} \mathcal{L S}(G)$.

In the following we introduce a new class of graphs which we will call by $s$-chorded cycle graphs. The $s$-chorded cycle graphs give rise to huge classes of inequalities generalizing the Kuratowski inequalities. In this section all sums of integers representing nodes of graphs $G=(V, E)$, which are greater than $n=|V|$ are to be taken modulo $n$.

Definition 2. For $s, n, r, t \in \mathbb{N}, s \geq 2, n=s t+r, 0 \leq r<s$, the $s$-chorded cycle graph $G_{(n, s, t)}=(V, E)$ is defined via

$$
\begin{aligned}
& V=\{1,2, \ldots, n\} \text { and } \\
& E=C_{n} \cup D_{n}, \quad \text { where }
\end{aligned}
$$

$C_{n}$ is a cycle of length $n, \quad C_{n}=\{(i, i+1) \mid i=1, \ldots, n\}$ and $D_{n}$ is the set of $s$-chords of $C_{n}, \quad D_{n}=\{(i, i+s) \mid i=1, \ldots, n\}$.

An $s$-chorded cycle graph gives rise to the definition of the corresponding $s$ chorded cycle inequality. In [M94b], it is investigated for which values of $s, t$ and $n$ the $s$-chorded cycle inequality induced by $G_{(n, s, t)}$ is valid, resp. facet-defining for $\mathcal{P} \mathcal{L S}\left(G_{(n, s, t)}\right)$.

Theorem 3 [M94b]. Let $G_{(n, s, t)}=(V, E)$ be a s-chorded cycle graph, i.e. $n=$ $s t+r$ for $s, t, r \in \mathbb{N}$ and $r \in\{0,1, \ldots, s-1\}$. We define 


$$
c_{e}= \begin{cases}n-2 t-s, & \text { if } x \in C_{n} \\ 1, & \text { if } x \in D_{n}\end{cases}
$$

and $c(E):=\sum_{e \in E} c_{e}$. If $n-2 t-s>0$, the $s$-chorded cycle inequality

$$
(n-2 t-s) x\left(C_{n}\right)+x\left(D_{n}\right) \leq c(E)-(n-2 t)
$$

is valid for $\mathcal{P} \mathcal{L S}\left(G_{(n, s, t)}\right)$ if and only if $(t \geq 3, s \geq 3)$ or $(t=2, s \geq 3, r \geq 2)$. Moreover, if the $s$-chorded cycle inequality is valid, then it is facet-defining for $\mathcal{P} \mathcal{L S}\left(G_{(n, s, t)}\right)$ if $r>0$.

Corollary 4 [M94b]. Let $G_{(n, s, t)}$ be an s-chorded cycle graph, which is a subgraph of $G$. If

$$
t=2 \text {, or } s=3 \text {, or } r \geq\left\lfloor\frac{s}{2}\right\rfloor
$$

and the corresponding s-chorded cycle inequality corresponding to $G_{(n, s, t)}$ is facet-defining for $G_{(n, s, t)}$, then the s-chorded cycle inequality is facet-defining for $\mathcal{P} \mathcal{L S}(G)$.

For $n=5$ the 2-chorded cycle inequality is identical to the Kuratowski inequality for $K_{5}$. So, the general $s$-chorded cycle inequalities are generalizations of the Kuratowski inequality for $K_{5}$. For the special case that $n=2 k+1$ and $s=k$, the $s$-chorded cycle graphs give rise to another kind of inequality, the odd $n$-ladder inequality, which is an alternative generalization of the $K_{5}$-inequality.

Theorem 5 [M94b]. If $G$ contains the $k$-chorded cycle graph $G_{(2 k+1, k, 2)}=(V, E)$ on $2 k+1$ nodes with $E=C_{2 k+1} \cup D_{2 k+1}, k \in \mathbb{N}, k \geq 2$, then the odd n-ladder inequality

$$
(2 k-3) x\left(C_{2 k+1}\right)+x\left(D_{2 k+1}\right) \leq(2 k-1)^{2}
$$

is facet-defining for $\mathcal{P} \mathcal{L S}\left(G_{(2 k+1, k, 2)}\right)$ and for $\mathcal{P} \mathcal{L S}(G)$.

In the special case $s=k$ and $n=2 k$, the $k$-chorded cycle graphs $G_{(2 k, k, 2)}$ contain multiple edges. If we take each diagonal only once, we obtain a Möbiusladder, which gives rise to an inequality which is a generalization of Kuratowski's $K_{3,3}$ inequality.

Definition 6. For $k \in \mathbb{N}, k \geq 3$, we define the (even) Möbius-ladder to be the graph $G_{M}=(V, E)$ with

$$
\begin{array}{ll}
V=\{1,2, \ldots, 2 k\} & \text { and } \\
E=C_{2 k} \cup D_{k}, & \text { where }
\end{array}
$$

$C_{2 k}$ is a cycle of length $2 k$,

$C_{2 k}=\{(i, i+1) \mid i=1, \ldots, 2 k\}$

$D_{k}$ is the set of longest chords of $C_{2 k}$,

$D_{k}=\{(i, i+k) \mid i=1,2, \ldots, k\}$. 
Theorem 7 [M94b]. For the Möbius-ladder graph $G_{M}=(V, E)$ with $E=C_{2 k} U$ $D_{k}$ and $k \geq 3$ the Möbius-ladder inequality

$$
(k-2) x\left(C_{2 k}\right)+x\left(D_{k}\right) \leq 2(k-1)^{2}
$$

is facet-defining for $\mathcal{P} \mathcal{L} S\left(G_{M}\right)$. Moreover, the Möbius ladder inequality is facetdefining for $\mathcal{P} \mathcal{L S}(G)$, whenever $G_{M}$ is a subgraph of $G$.

A complete overview of the currently known structure of the planar subgraph polytope can be found in [M94a].

\section{The $k$-connected Subgraph Polytope $k-\mathcal{N C S}(G)$}

Given a graph $G_{0}=\left(V_{0}, E_{0}\right)$, we are interested in the set of all $k$-connected spanning subgraphs of $G_{0}$. If $G_{0}$ is not $k$-connected, the set of all $k$-connected subgraphs is the empty set. In this case we are interested in augmenting $G_{0}$ in order to reach $k$-connectivity. We associate the $k$-connected (spanning) subgraph polytope to a $k$-connected graph $G=(V, E)$, which contains $G_{0}$ as a subgraph, e.g. $G=K_{n}$ with $n=\left|V_{0}\right|$. For each $k$-connected subgraph $K=\left(V^{\prime}, F\right)$ of $G$ we define its incidence vector $\chi^{K} \in \mathbb{R}^{E}$ by setting $\chi_{e}^{K}=1$ if $e \in F$ and $\chi_{e}^{K}=0$ if $e \notin F$. The $k$-connected (spanning) subgraph polytope $k-\mathcal{N C S}(G)$ is defined as the convex hull over all incidence vectors of $k$-connected spanning subgraphs of $G$. In order to solve the minimum $k$-connected subgraph problem for a given $k$-connected graph $G_{0}=\left(V_{0}, E_{0}\right)$, we choose $G:=G_{0}$ and define the weight $c_{e}$ for an edge to be 1 . The problem of determining a $k$-connected subgraph of $G$ with the minimum number of edges, can be formulated as the linear program $\min \left\{c^{T} x \mid x \in k-\mathcal{N C S}(G)\right\}$. If we like to solve the $k$-augmentation problem for a given graph $G_{0}$, we choose $G=(V, E):=K_{n}$, define the weight $c_{e}$ for an edge to be $-M$, if $e \in E_{0}$ and 1 if $e \notin E_{0}$, where $M=\left|E \backslash E_{0}\right|+1$, and solve $\min \left\{c^{T} x \mid x \in k-\mathcal{N C S}(G)\right\}$.

The $k$-connected subgraph polytope was already studied by Stoer in a more general form [S92]. For $k=2$, the computational results in [S92] are promising.

Theorem 8 [S92]. The integer points of $k-\mathcal{N C S}(G)$ are characterized by the following system of inequalities:

$$
\begin{array}{ll}
0 \leq x_{e} \leq 1, & \text { for all } e \in E \\
x\left(\delta_{G-Y}(W)\right) \geq 1, & \text { for all } Y \subseteq V,|Y|=k-1, W \subseteq V \backslash Y \\
x_{e} \text { integral, } & \text { for all } e \in E
\end{array}
$$

The inequalities (5) essentially say that if a node set $Y \subseteq V$ of size $k-1$ is removed, the resulting graph must still be connected. 


\section{$4 \quad$ Intersecting $\mathcal{P} \mathcal{L S}(G)$ and $k-\mathcal{N C S}(G)$}

In this section we are interested in the integer points contained in the polytope

$$
k-\mathcal{N} \mathcal{C P} \mathcal{L S}(G)=k-\mathcal{N C S}(G) \cap \mathcal{P} \mathcal{L} \mathcal{S}(G)
$$

We already gave a system of inequalities characterizing the integer points for both polytopes. So, the integer points of the new polytope $k-\mathcal{N C P} \mathcal{L S}(G)$ is defined by the system of inequalities given by (1), (2), (3) and (5).

By choosing $G:=G_{0}=\left(V_{0}, E_{0}\right)$ and setting $c_{e}=1$ for all edges, or by choosing $G:=K_{n}$ and setting

$$
c_{e}= \begin{cases}1, & \text { if } e \in E_{0} \text { and } \\ -M, & \text { if } e \notin E_{0},\end{cases}
$$

where $M^{\prime}:=\left|E_{0}\right|+1$, we can formulate the maximum planar $k$-connected subgraph problem for a given graph $G_{0}$ by $\max \left\{c^{T} x \mid x \in k-\mathcal{N} \mathcal{C P} \mathcal{L S}(G)\right\}$.

The planar $k$-augmentation problem is obtained by choosing $G$ to be the complete graph on $\left|V_{0}\right|$ vertices, and setting

$$
c_{e}= \begin{cases}M^{\prime}, & \text { if } e \in E_{0} \text { and } \\ -1, & \text { if } e \notin E_{0},\end{cases}
$$

where $M^{\prime}=\left|E \backslash E_{0}\right|+1$. Now, the planar $k$-augmentation problem can also be written as $\max \left\{c^{T} x \mid x \in k-\mathcal{N} \mathcal{C P} \mathcal{L} \mathcal{S}(G)\right\}$.

For both problems we introduced big $M$ 's in order to guarantee that either none of the forbidden edges of $E \backslash E_{0}$ or all required edges in $E_{0}$ will be contained in the optimum solution. But having big $M$ 's as objective coefficients may lead to numerical difficulties during computation. This motivates in a natural way the definition of the minimum planarizing $k$-augmentation problem, which is given by setting $G:=K_{n}$ and

$$
c_{e}= \begin{cases}1, & \text { if } e \in E_{0} \text { and } \\ -1, & \text { if } e \notin E_{0} .\end{cases}
$$

Maximizing $c^{T} x$ leads to taking as many edges of $G_{0}$ as possible and as few edges of $G-G_{0}$ as possible. This way, the difference between the given graph $G$ and the new graph $H$ will be minimized.

Obviously, the number of edges we have to delete from $G_{0}$ plus the number of edges we have to add to $G_{0}$ is given by $c^{T}\left(\chi^{G_{0}}-x\right)$, where $\chi^{G_{0}}$ denotes the incidence vector of $G_{0}$. Hence, also this problem can be written as $\max \left\{c^{T} x \mid\right.$ $x \in k-\mathcal{N} \mathcal{C P} \mathcal{L S}(G)\}$.

Since choosing $G:=K_{n}$ increases the size of the original graph a lot and the set of edges which is never in any augmentation is huge, we may like to choose as $G$ an arbitrary graph containing $G_{0}$ and a small subset of edges which are 
likely to be in the optimum solution. We can also give more relative emphasis to $k$-connectivity than to planarity and vice versa by setting

$$
c_{e}= \begin{cases}a, & \text { if } e \in E_{0} \text { and } \\ -b, & \text { if } e \notin E_{0}\end{cases}
$$

for arbitrary $a, b>0$.

If we wish to consider problem (1) and the degree-constrained versions of the problems (2), (3) and (4), we just have to add the degree constraints

$$
x \delta(v)) \leq D(v) \quad \text { for all } v \in V
$$

to the integer linear programming formulation, and the corresponding polytope becomes the intersection of three polytopes, namely the planar subgraph polytope, the $k$-connected subgraph polytope and the "degree-constrained subgraph polytope".

\section{The Algorithm}

In [JM94] we give a branch and cut algorithm for the maximum planar subgraph problem using facet-defining inequalities for $\mathcal{P} \mathcal{L S}(G)$ as cutting planes. In a cutting plane algorithm, a sequence of relaxations is solved by linear programming. After the solution $x$ of some relaxation is found, we must be able to check whether $x$ is the incidence vector of a planar subgraph (in which case we have solved the problem) or whether any of the known facet-defining inequalities are violated by $x$. If no such inequalities can be found, we cannot tighten the relaxation and have to resort to branching, otherwise we tighten the relaxation by all facet-defining inequalities violated by $x$ which we can find. Then the new relaxation is solved, etc. The process of finding violated inequalities (if possible) is called "separation" or "cutting plane generation".

The cutting plane generation as well as the lower bound heuristic are based on a planarity testing algorithm of Hopcroft and Tarjan [HT74]. At the beginning we solve the Linear Program (LP) consisting of the trivial inequalities $x_{e} \geq 0$, $x_{e} \leq 1$ and the inequality $x(E) \leq 3|V|-6$. Let $x$ be an LP-solution produced in the cutting plane procedure applied in some node of the enumeration tree. For $0 \leq \varepsilon \leq 1$ we define $E_{\varepsilon}=\left\{e \in E \mid x_{e} \geq 1-\varepsilon\right\}$ and consider $G_{\varepsilon}=\left(V, E_{\varepsilon}\right)$. For the unweighted graph $G_{\varepsilon}$ the linear planarity testing algorithm of Hopcroft and Tarjan is called. The algorithm stops if it finds an edge set $F$ which is not planar. In case the inequality $x(F) \leq|F|-1$ is violated, we reduce it to a facet-defining inequality before we add it to the constraints of the current LP. We also use a heuristic which searches for violated Euler-inequalities and inequalities given by some classes of $s$-chorded cycle graphs.

Although the vectors $x$ coming up as solutions of LP-relaxations in the above outlined process have fractional components in general, they are often useful to obtain information on how a high-valued planar subgraph might look like. We 
exploit this idea with a greedy type heuristic with respect to the solution values of the edges. Starting from the empty graph, a planar subgraph is constructed by adding the edges in order of decreasing values if they do not destroy planarity. So, in addition to the upper bounds $w^{T} x$ on the value of a maximum planar subgraph, we also obtain a lower bound $w^{T} \bar{x}$ from the planar subgraph incidence vector $\bar{x}$ derived heuristically from $x$.

In our computational experiments for the maximum planar subgraph problem we solved several problems from the literature to optimality. For solving the degree-constrained maximum planar subgraph problem, we add the inequalities (7) to the LP. In order to solve, for example, the maximum planar 2-connected subgraph problem, the planar 2-augmentation problem or the minimum planarizing 2-augmentation problem, we suggest to use the above algorithm extended by the use of the inequalities corresponding to the $k$-connected subgraph polytope given in (5). For $k=2$, the inequalities reduce to

$$
x\left(\delta_{G-\left\{v_{0}\right\}}(W)\right) \geq 1 \quad \text { for all } v_{0} \in V, W \subseteq V \backslash\left\{v_{0}\right\}
$$

Given an LP-solution $y$ produced in the cutting plane procedure, we are able to give a node $v_{0} \in V$ and a set $W, W \subseteq V \backslash\left\{v_{0}\right\}$ violating inequality (8) or guaranteeing that all the inequalities in (8) are satisfied by $y$. This can be done in polynomial time by the following separation routine. For all nodes $v_{0} \in V$ construct the graph $G^{\prime}:=G-\left\{v_{0}\right\}=\left(V^{\prime}, E^{\prime}\right)$ and search for the minimum cut in $G^{\prime}$ with edge values $y_{e}$ for all $e \in E^{\prime}$. Let $z$ be the value of this minimum cut. If $z>1$, all inequalities of the type (8) for the specific node $v_{0}$ are satisfied. Otherwise, the inequality $x\left(\delta_{G-\left\{v_{0}\right\}}(W)\right) \geq 1$ is violated for the set $W$ determined by the minimum cut $\delta(W)$.

\section{Computational Experiments}

Up to now we have only implemented the easiest case in which the planar subgraph polytope is intersected with the degree-constrained subgraph polytope. The other cases require a much higher implementation effort which we plan to carry out in the future.

Our computational experiments were run on a SUN SPARCstation 10/41. The following table shows the results for solving the maximum planar subgraph problem and the degree-constrained maximum planar subgraph problem for several graphs occuring in the literature of automatic graph drawing. The columns from left to right display the problem name, resp. the origin of the problem plus the origin of the published computational result, if it appeared elsewhere, the number of nodes, the number of edges, the value of the optimal solution found by our algorithm, and the CPU times in seconds (Fractions of seconds are not shown) for the maximum planar subgraph problem and for the degreeconstrained maximum planar subgraph problem.

In the cases we tested, with one exception, it turned out that the degreeconstrained version of the maximum planar subgraph problem is easier. The 
Table 1. Results for the (degree-constrained) maximum planar subgraph problem

\begin{tabular}{|r|r|r|r|r|r|r|}
\hline Author & \#Nod & \#Edg & Sol-MPS & Tim-MPS & Sol-DCMPS & Tim-DCMPS \\
\hline [JTS89] & 10 & 22 & 20 & 0 & 18 & 0 \\
{$[$ K92] } & 45 & 85 & 82 & 5 & 61 & 1 \\
{$[$ [C92] } & 60 & 166 & 165 & 4 & 120 & 94 \\
\hline [BGHS2] & 11 & 21 & 20 & 0 & 17 & 0 \\
{$[$ E93] } & 20 & 30 & 28 & 1 & 28 & 0 \\
{$[$ H93] } & 34 & 45 & 43 & 1 & 35 & 1 \\
{$[$ EM94] } & 38 & 73 & 66 & 7972 & 63 & 434 \\
[TBB88,STT81] & 43 & 62 & 58 & 31 & 53 & 0 \\
{$[$ H93] } & 46 & 64 & 62 & 1 & 59 & 1 \\
{$[$ H93] } & 48 & 69 & 64 & 327 & 56 & 0 \\
\hline
\end{tabular}

optimum solution of the "competition graph" given by Eades and Marks in the Graph Drawing Competition'94 [EM94] is found in a few seconds, but it took us about 2 hours to prove it, in the degree-constrained version, however, it took only 7 minutes.

\section{References}

[BGHS92] Beck, H.K.B., H.-P. Galil, R. Henkel, and E. Sedlmayr: Chemistry in circumstellar shells, I. Chromospheric radiation fields and dust formation in optically thin shells of M-giants. Astron. Astrophys. 265 (1992) 626-642

[C92] Cimikowski, R.J.: An Empirical Analysis of Graph Planarization Heuristics. Computer Science Dept., Montana State Univ. (1992)

[E93] Eades, P.: Personal communication (1993)

[EM94] Eades, P. and J. Marks: Personal communication (1994)

[F92] Frank, A.: Augmenting graphs to meet edge-connectivity requirements. SIAM J. Discr. Math. 5 (1992) 25-53

[H93] Himsolt, M.: Konzeption und Implementierung von Grapheneditoren. Dissertation, Universität Passau (1993)

[HR91] Hsu, T.-S. and V. Ramachandran: A linear time algorithm for triconnectivity augmentation. Proc. 32th Annual Symp. on Found. of Comp. Science, Puerto Rico (1991) 548-559

[HT74] Hopcroft, J., and R.E. Tarjan: Efficient planarity testing. J. ACM 21 (1974) $549-568$

[JM93] Jünger, M. and P. Mutzel: Solving the Maximum Planar Subgraph Problem by Branch and Cut. Proceedings of the 3rd International Conference on Integer Programming and Combinatorial Optimization (IPCO 3), Erice (1993) 479-492

[JM94] Jünger, M. and P. Mutzel: Maximum planar subgraphs and nice embeddings: Practical layout tools. to appear in Algorithmica, special issue on Graph Drawing, Edit. by G. Di Battista und R. Tamassia (1994)

[JTS89] Jayakumar, R., K. Thulasiraman and M.N.S. Swamy: $\mathrm{O}\left(n^{2}\right)$ Algorithms for Graph Planarization. IEEE Trans. on Computer-aided Design 8 (1989) $257-267$ 
[K92] Kant, G.: An $\mathrm{O}\left(n^{2}\right)$ Maximal Planarization Algorithm based on PQ-trees. Technical Report, RUU-CS-92-03, Dept. of Computer Science, Utrecht University (1992)

[K93] Kant, G.: Algorithms for Drawing Planar Graphs. Ph.D.-Thesis, Utrecht University (1993)

[M94a] Mutzel, P.: The Maximum Planar Subgraph Problem. Dissertation, Universität Köln (1994)

[M94b] Mutzel, P.: s-Chorded Cycle Graphs and their Relation to the Planar Subgraph Polytope. Technical Report No. 94-161, Angewandte Mathematik und Informatik, Universität zu Köln (1994)

[RG77] Rosenthal, A. and A. Goldner: Smallest augmentation to biconnect a graph SIAM J. on Computing 6 (1977) 55-66

[S92] Stoer, M.: Design of Survivable Networks. Lecture Notes in Mathematics, Springer-Verlag, Berlin (1992)

[STT81] Sugiyama, K., S. Tagawa, and M. Toda: Methods for Visual Understanding of Hierarchical Systems. IEEE Trans. on Systems, Man and Cybernetics, SMC-11, 2 (1981) 109-125

[TBB88] Tamassia, R.: On embedding a graph in the grid with the minimum number of bends. SIAM J. Comput. 16 (1987) 421-444

[TBB88] Tamassia, R., G. Di Battista, and C. Batini: Automatic graph drawing and readability of diagrams. IEEE Transactions on Systems, Man and Cybernetics 18 (1988) 61-79 